\title{
Anabases
}

ANABASES Traditions et réceptions de l'Antiquité

$10 \mid 2009$

Varia

\section{Maxima quaestio et semper incerta}

\section{Philippe Borgeaud}

\section{OpenEdition}

Journals

Édition électronique

URL : http://journals.openedition.org/anabases/554

DOI : 10.4000/anabases.554

ISSN : 2256-9421

\section{Éditeur}

E.R.A.S.M.E.

\section{Édition imprimée}

Date de publication : 1 octobre 2009

Pagination : 29-36

ISSN : 1774-4296

\section{Référence électronique}

Philippe Borgeaud, « Maxima quaestio et semper incerta », Anabases [En ligne], 10 | 2009, mis en ligne le 01 octobre 2012, consulté le 20 octobre 2019. URL : http://journals.openedition.org/anabases/554 ; DOI : 10.4000/anabases.554 
Anabases 10 (2009), p. 29-36.

\title{
Maxima quaestio et semper incerta
}

\author{
Philippe Borgeaud
}

Car un dieu toujours reconnaît un autre dieu, fût-il de ceux qui vivent aux confins du monde Homère, Odyssée, 5, 79-80

MAXIMA QUAESTIO ET SEMPER INCERTA, «très grande question, et toujours ouverte», disait Pline l'Ancien posant la question de la fides, du "croire ${ }^{1} »$. Au fond, j'ai l'impression qu'il ne saurait en aller autrement quand on pose la question des filiations. La filiation est forcément une affaire peu claire. Qu'on soit arrivé où l'on est aujourd'hui, à la suite d'un itinéraire compliqué mais précis, cela est indéniable. Mais aussitôt qu'on essaie, rétrospectivement, de reconstruire le chemin parcouru, c'est un labyrinthe qui surgit, un univers éclaté, dispersé comme une nébuleuse que la mémoire, privée d'Ariane et de son fil, ne peut recomposer une fois pour toutes. Voici donc ma version d'aujourd'hui, un exercice, une interprétation parmi d'autres possibles.

Qui m'a appris à travailler? L'ennui d'abord, le calme plat. Arraché, accidentellement séparé d'avec un milieu de poètes et d'intellectuels plus ou moins marginaux et révolutionnaires où circulaient mes parents, je me suis retrouvé pour quinze années, jusqu'à l'âge de dix-neuf ans, dans une campagne magnifique au pied des Alpes, recueilli avec la plus grande sollicitude, tendresse et sévérité par le père de ma mère, un ingénieur retraité, franc-maçon, vouant un culte à Einstein, et son autre fille, devenue veuve, curieux mélange de Madame Bovary et de Lady Chatterley. Je fus élevé (plus ou moins bien élevé) dans un collège voisin tenu par des chanoines férus de mystique

1 Pline l'Ancien, Histoire naturelle XXVIII, III, trad. A. Ernout, Collection des Universités de France, Paris, Les Belles-Lettres. 
et tourmentés par le sexe, savants en philosophie thomiste, en grec et en latin, au pied d'un grand rocher triste et humide. J'appris à lire, à beaucoup lire. Villon, Montaigne, Pascal, Baudelaire, Rimbaud et (en cachette) Henri Michaux et le Céline du Voyage au bout de la nuit, à côté d'Apollinaire et de Breton.

Cette existence en exil studieux fut ponctuée d'échanges et de retrouvailles intenses et éphémères. Je me souviens du train (deux heures de trajet solitaire, collé à la vitre) qui me ramenait, de temps en temps, trop peu souvent, vers quelques jours d'effervescence. Je me souviens aussi d'une collection de timbres abandonnée par mon père, avec beaucoup de timbres reçus de pays africains aujourd'hui disparus ou rebaptisés, visités vers 1920 par un parent aventurier, et aussi beaucoup de timbres allemands oblitérés à Rostock, Halle-an-der Saale et Berlin entre 1939 et 1944, des villes où mon père avait étudié, quelques années avant ma naissance (je suis né en 1946), le vieil-irlandais, le sanscrit, l'avestique et la grammaire comparée, avec une bourse de la Alexander von Humboldt Stiftung. Il m'avait transmis cette collection que je m'efforçai de prolonger, pendant quelques années, avec les timbres décollés de lettres qu'il m'envoyait du ProcheOrient. C'est ainsi que je découvrais, à distance, les ruines et l'histoire de l'Égypte, de Damas et d'Alep, de Jérusalem et du Liban. Un colis très important me fut enfin envoyé depuis Le Caire, comprenant une histoire de l'art universelle en deux volumes (que je possède encore et dont je peux "voir» en pensée quasiment chaque illustration), le livre de C.-W. Céram, Des Dieux, des Tombeaux, des Savants, ainsi que Je lis les hiéroglyphes de Jean Capart et, en anglais, une langue que je n'apprenais pas au collège, l'étude très savante de Parker sur le calendrier égyptien (que je n'ai jamais vraiment lue). Tout cela était accompagné d'une traduction inédite du fameux "cantique» des Danaïdes, tiré des Suppliantes d'Eschyle, et d'une enquête pour moi incompréhensible, mais fascinante, sur Dieux indo-européens et dieux phéniciens. Qui sont Agénor et Téléphassa, souverains de $\operatorname{Tyr}^{2}$ ?

Quand je voyais (rarement) mon père, il me faisait lire en allemand des légendes africaines rapportées par Frobenius. Quand plus tard, après bien des errances et beaucoup de misère, il trouva un poste à l'Université d'Ottawa comme professeur de latin et de grec, et que je devenais de mon côté, à l'Université de Genève, étudiant en grec, en littérature française et en histoire des religions antiques, il n'a cessé de m'envoyer des lettres enthousiastes et tourmentées, emplies d'échos de sa vie affective et de réflexions étymologiques et poétiques. Il voulait que j'apprenne le sanscrit. Il me fit déchiffrer, tant bien que mal, en m'en expliquant chaque mot et chaque forme grammaticale, l'hymne védique à l'Un. Quand j'eus terminé la rédaction de ma thèse de doctorat, Recherches sur le dieu Pan, il en relut les épreuves pour vérifier l'exactitude des passages en grec, et me fit cadeau d'un commentaire sur l'étymologie de Pan qu'il avait rédigé avec sa

2 Conférence publique donnée à l'Amphithéâtre de l'Université de Damas le jeudi 7 avril 1960 par le Dr. Willy Borgeaud, Professeur visiteur à l'Université de Damas, Année Académique 1959-1960, Presses de l'Université de Damas. 
seule mémoire, sur une île grecque, loin de toute bibliothèque. Ce texte figure comme appendice dans l'édition de mes Recherches publiée par l'Institut Suisse de Rome.

Pour résumer la chose en quelques mots, ce que je lui dois, en ce qui concerne mon métier, ce n'est certainement pas la méthode, ni la pratique des langues. Du côté de celles-ci je me contente du minimum et, du côté de la méthode, j’ai développé très vite (sous l'influence de mes professeurs), une certaine méfiance pour les origines étymologiques.

Ce que je lui dois c'est la révélation, très tôt, du vaste monde et d'un ensemble de textes merveilleux dont on ne parle guère à l'école. L'émerveillement face à la "matière" du mythe, qui ne m'a plus lâché. Une matière foisonnante et plurielle valant «en soi », indépendamment de la méthode.

Je n'avais bien sûr, moi-même, aucune méthode. Mais j'étais littéralement fasciné par ces paroles issues d'Afrique ou d'Asie, de Rome ou de Grèce. Cet émerveillement grandit en lisant, adolescent, quelques livres laissés sur une table où mon père travaillait parfois et où je passais, en compagnie de ma grand-mère, une petite paysanne veuve et calviniste parfaitement inculte, tentée par les Témoins de Jéhova, de longues après-midis de vacances dans un salon sombre et feutré, hanté par le son monotone et engourdissant d'un vieux morbier: les Essais sur le Bouddhisme Zen de Suzuki, une édition et traduction des Upanishad en fascicules, sous la direction de Louis Renou, Horace et les Curiaces de Dumézil, l'Introduction à l'essence de la mythologie de Karl Kérényi et C.G. Jung, le Rameau d'or de James George Frazer dans la version française abrégée par Lady Frazer, la Mythologie universelle et La Genèse des mythes d'Alexander Krappe, enfin et surtout le Traité d'histoire des religions de Mircea Eliade. J'avais alors 16 et 17 ans.

Ce qui finit par retenir mon attention (cela s'explique probablement...), ce furent les dieux de l'Égypte ancienne (un domaine éloigné des préoccupations indo-européennes de mon père). Je décidais d'apprendre les hiéroglyphes. Pour cela, je me fis offrir une vieille grammaire égyptienne du XIX ${ }^{\mathrm{e}}$ siècle, et on me mit en contact épistolaire avec une assistante de la chaire genevoise d'égyptologie (une comtesse, très jeune et très belle). Elle accepta de corriger quelques exercices que je lui envoyais depuis ma campagne.

À l'Université, c'est tout naturellement l'égyptologie que je choisis comme branche principale. J'en suivis les cours durant deux ans, parvenant plus ou moins à lire le Conte du Naufragé. Mais je fus très vite déçu par un enseignement exclusivement centré sur la grammaire, l'histoire et l'archéologie, sans ouverture en direction de la pensée ou des constructions imaginaires.

Les cours de Jean Rudhardt, qui venait d'être nommé professeur d'histoire des religions dans le département des sciences de l'Antiquité, m'enchantèrent. Avec lui, je pouvais reprendre, sans exaltation naïve, la matière du mythe (une matière qu'il 
venait de découvrir, lui aussi ${ }^{3}$ ). Mais il fallait passer par le grec. Je refis donc du grec et du latin. Avec Rudhardt, je fis aussi le rude apprentissage d'une nécessaire et salutaire prudence. Rudhardt n'aimait pas les théories. Il exigeait que nous revenions aux sources, et que nous nous y tenions. Pas moyen, chez lui, de citer un moderne comme autorité. Il n'y avait d'autorité que celle des sources anciennes... et la sienne. Au fond, comme professeur, Rudhardt fut un parfait déconstructionniste. Il fit s'écrouler toutes les bases aléatoires de mes enthousiasmes frazériens, duméziliens ou éliadiens. Mais il fit cela en encourageant cependant la plus grande liberté. Quand je lui dis, au printemps 1968, que j'aurais l'occasion de me rendre au Mexique en mai-juin et que cela me ferait manquer un mois de ses cours, il me dit sans hésiter que j'y apprendrais plus qu'en l'écoutant parler. Olivier Reverdin, qui enseignait le grec, m'encouragea lui aussi: il me prêta la précieuse traduction de l'Histoire générale des choses de la Nouvelle Espagne de Bernardino de Sahagún par D. Jourdanet et R. Siméon (Paris 1880). Cette lecture, autant que le voyage, fut déterminante. Elle déclencha mon intérêt pour l'ethnologie, et pour l'étude des chroniqueurs et des missionnaires considérés comme des historiens des religions avant la lettre. De là à découvrir le regard des pères de l'Église sur les religions polythéistes, il n'y avait qu'un pas, que je n'allais pas tarder à franchir.

Quand je revins à Genève en octobre 1968, Jean Starobinski (mon professeur préféré de littérature française, à qui je devais la découverte, elle aussi ethnologique, du Quart Livre de Rabelais) me demanda ce que j'avais fait depuis le printemps. Il fut enchanté de ma non-participation aux "événements", mais sa réaction (semblable à celle de Jeanne Hersch d'ailleurs, autre ténor de l'École genevoise), m'a laissé un goût amer, car la découverte de l'altérité, finalement, m'avait empêché d'être acteur de ma propre histoire. J'étais, une fois de plus, «hors du coup».

Lors de la soutenance de mon mémoire de licence en juillet 1970, André Hurst, assistant d'Olivier Reverdin, me suggéra de prendre contact avec un savant dont (scandaleusement) j'ignorais tout: Marcel Detienne. Je pris rendez-vous par téléphone, et me rendis à Paris, Place de la Sorbonne où (étant donné la bibliographie parcourue la veille) je m'attendais à rencontrer un vieillard. Quelle surprise! Et quel enthousiasme communicatif, aussitôt. Un monde s'ouvrait d'un coup, généreux, où la philologie rejoignait, sans scrupule, l'anthropologie.

3 Il ne s'y intéressait pas encore dans sa thèse, Notions fondamentales de la pensée religieuse et actes constitutifs du culte dans la Grèce classique, parue à Genève, chez Droz, en 1958 et rééditée à Paris, chez Picard, en 1992. La chose change avec "Une approche de la pensée mythique: le mythe considéré comme un langage”, Studia philosophica 26 (1966), p. 208-237 (republié dans Du mythe, de la religion grecque et de la compréhension d'autrui, Genève, 1981, Cahiers Vilfredo Pareto: revue européenne des sciences sociales, tome XIX, $\left.\mathrm{n}^{\circ} 58\right)$. 
Jean Rudhardt m'avait appris à lire et à comparer, avec une prudence toutefois, voire une rétention du savoir très genevoises, un peu paralysantes ${ }^{4}$. Quand j'arrivais à Chicago pour une année d'étude "at large» auprès d'Eliade et de Jonathan Smith, en septembre 1971, j'avais la plus grande peine à oser émettre un avis tranché sur ce qui était au fond ma spécialité, la religion grecque. Si l'on me demandait ce qu'étaient les mystères d'Eleusis, je répondais, un peu effrayé, qu'on n'en savait trop rien. À Genève, on ne m'avait jamais suggéré de publier la moindre chose, et on m'avait en quelque sorte dissuadé d'avoir des opinions. J'étais censé préparer ma thèse. Cela suffisait. À peine débarqué à Chicago, je fus plongé dans une atmosphère dont j'avais entrevu l'analogue à Paris en découvrant, à travers Detienne, l'école de Jean-Pierre Vernant. Je fus dès lors contraint d'avoir des idées et aussi d'oser les écrire.

À la différence de Paris cependant il n'y avait pas, à Chicago, une «école» où chacun (en ce temps-là) contribuait (ou pensait contribuer) à un édifice commun. Mais une curiosité décidément plurielle, sans limite. On pouvait y citer Lévi-Strauss à côté d'Eliade, Walter Burkert avec Vernant ou Detienne.

Peu intéressé par mon projet de thèse sur le dieu Pan, un projet exclusivement grec, Eliade me demanda si j'avais d'autres intérêts. Quand je lui dis que je travaillais depuis quelque temps, avec quelques amis, en dehors de l'université, sur le motif du labyrinthe, il me proposa aussitôt de préparer un texte pour sa revue, History of Religions. Je n'oublierai jamais cette perche tendue à un débutant, l'encourageant à écrire son premier article «scientifique». D'Eliade je retiendrai aussi le premier séminaire auquel je participai à Chicago : il entra avec une petite valise emplie de livres qu'il venait de découvrir (parmi eux: I Benandanti de Carlo Ginzburg). Il expliqua, d'une manière très peu formelle, aux quelques élus autorisés à travailler avec lui (dont Bruce Lincoln, David Carrasco et Georges Tissot), quel serait le thème de l'année avant de distribuer les rôles en fonction des spécialités de chaque étudiant. Ensuite, ses interventions furent limitées aux questions et commentaires adressés à nos exposés. Ce qu'il nous donnait de plus intéressant, au fond, c'était son attention, son intérêt. Un intérêt confirmé quand il nous recevait, régulièrement, dans son bureau situé à quelques pas de sa maison. Sa prodigieuse érudition savait toujours trouver la petite référence insoupçonnée qui nous précipitait vers de nouvelles pistes.

Les bases du métier d'historien des religions, ce n'est pas à Eliade que je les dois, mais à son collaborateur Jonathan Smith, auteur d'une thèse inédite sur le Rameau d'or, dont il avait lu toutes les sources. Jonathan Smith donnait le cours d'introduction à l'histoire des religions, une discipline, comme il nous le dit en commençant, «à la recherche de sa propre définition». Il nous faisait lire et analyser, parmi bien d'autres

4 Dans l'école de Marcel Raymond, de Jean Rousset et de Jean STARobinski, l'encouragement à écrire fut relativement exceptionnel : cf. le témoignage drôle et instructif de Luc WEIBEL, Une thèse pour rien. La comédie du savoir, Paris, Le Passage Paris-New York Éditions, 2003. 
choses, des mythes maori et winnebago. Il me fit découvrir aussi les livres de Victor Turner (notamment The Ritual Process). Chez Victor Turner, un mercredi soir où le maître recevait ses disciples, à l'occasion d'une présentation de L'Homme Nu de LéviStrauss, je rencontrai pour la première fois James Redfield qui préparait sa Tragédie $d^{\prime}$ Hector $^{5}$. Redfield, par des liens subtils nouant le grec à l'anthropologie, via le Yucatan où je rendis visite durant l'été 1979 au village de son enfance (Chan Kom, le «terrain » de son père Robert Redfield, pilier de l'anthropologie américaine) allait devenir un ami très proche. George Tissot, un camarade un peu gourou, me révélait, de son côté, les Mours des Sauvages Amériquains comparées aux Mours des Premiers Temps, du Père Joseph François Lafitau, qui servira de base à ma réflexion sur les mécanismes de la comparaison en histoire des religions ${ }^{6}$.

Après un second passage par le Mexique (en été 1972), je me rendis à Rome, à l'Institut suisse. Je désirais travailler avec Angelo Brelich, à la suggestion d'Eliade. Ce fut impossible, étant donné l'état de santé de Brelich. Mais à Rome j'ai connu l'école de Pettazzoni, et j'ai beaucoup erré, d'une bibliothèque à l'autre, beaucoup engrangé, beaucoup rêvé.

Rentré à Genève, comme assistant en histoire des religions antiques, j'ai continué d'être en relation avec Marcel Detienne et Jean-Pierre Vernant, tout en travaillant à ma thèse sur le dieu Pan, et en donnant des séminaires sur l'anthropologie religieuse, avec la bénédiction de Jean Rudhardt, qui venait à mes cours comme j'allais aux siens. J'ai en quelque sorte "participé» durant des années à la genèse de sa théorie du mythe et du sacré, et à ses premières recherches sur les Hymnes orphiques, comme il a su, par ses critiques redoutables, orienter mes propres explorations du côté du polythéisme, en m'incitant à ne pas rechercher l'«essence» du dieu Pan, mais à mettre Pan en contexte, en compagnie d'Hermès, de Dionysos, d'Artémis, de Déméter.

En 1979, après avoir soutenu et publié ma thèse, je suis retourné à Chicago, avec l'aide du Fonds national suisse, pour une recherche post-doctorale sur "Les compagnons de la Mère" (un projet à l'origine de mon intérêt pour celle que l'on nomme, parfois, Cybèle). Cette fois-ci, je décidai de fréquenter, d'abord, Arnaldo Momigliano. Il donnait, avec Edward Shils, un séminaire passionnant sur Ancient Judaism de Max Weber. Je découvris à la fois l'histoire de l'histoire, le judaïsme et l'importance de la période hellénistique.

Momigliano m'a fait lire Bachofen, tandis que Shils me signalait les notes de lecture de Marx sur le Mutterrecht. Je dois beaucoup à Momigliano, dont un compte

5 Nature and Culture in the Iliad: the Tragedy of Hector, Chicago, The University of Chicago Press 1975.

6 Cf. "Le problème du comparatisme en histoire des religions", in Gérald BerTHOUD et Giovanni Busino, La comparaison en sciences humaines et sociales, Revue Européenne des Sciences Sociales (Cahiers Vilfredo Pareto), t. XXIV, n ${ }^{\circ}$ 2, 1986, p. 59-75. 
rendu généreux de ma thèse fut probablement décisif pour ma nomination, en 1987, à la chaire d'histoire des religions à Genève.

Jean-Pierre Vernant, autre grand modèle, autre soutien, je l'ai connu non seulement à Paris, mais aussi sur son territoire valaisan, à La Sage, en compagnie de Maurice Olender. Il nous expliquait, au coin du feu, son expérience d'enseignant et de résistant à Toulouse. À travers lui, j'ai pu établir des échanges très fructueux entre Jean Rudhardt et Jean-Louis Durant, Pierre Ellinger, Nicole Loraux, Giulia Sissa, Stella Georgoudi. Il m'a appris à ne pas attribuer aux convictions scientifiques plus d'autorité qu'elles n'en méritent, à l'occasion d'une visite que je faisais au séminaire de Detienne une année avant la soutenance de ma thèse. Les disciples, qui n'étaient pas tous encore mes amis, me reprochaient en chœur, à propos de mon exposé sur Pan et la panique, de n'avoir point interprété ce dossier en fonction de la mètis, leur préoccupation de ce temps-là (deux ans après la parution du livre programmatique sur Les ruses de l'intelligence). Vernant leur fit remarquer que la mètis, après tout, ne pouvait pas et n'avait pas à être mise à toutes les sauces... et que d'autres thématiques pouvaient aussi s'avérer intéressantes.

Jean Rudhardt, le maitre proche, l'ami, m’a initié à l'expérience de l'altérité, de ce qu'il appelait «la compréhension d'autrui», avant que je ne prenne enfin et définitivement conscience, grâce à des séjours répétés du côté de Paris et Chicago, de ce qui oppose une démarche historienne et anthropologique à l'approche théologique et phénoménologique, celle d'Eliade. De cette séparation qui ne signifie pas que l'on renonce à l'émerveillement, je me suis expliqué dans un texte écrit à la demande (un peu naïve) de l'Institut National Genevois, où l'on découvre que des idéologies (nationalistes) pernicieuses peuvent se cacher derrière certaines pratiques lettrées apparemment innocentes ${ }^{7}$.

Je suis entré en mythologie en un temps où chaque livre de Dumézil, d'Eliade et de Lévi-Strauss était un événement. Le métier que j'ai appris et que j'aime encore est celui des historiens de l'Antiquité inspirés par la comparaison, des antiquisants ouverts à l'école sociologique française (celle de Marcel Mauss revisitée par Jean-Pierre Vernant, Pierre Vidal-Naquet et Marcel Detienne), ainsi qu'aux travaux essentiels de l'anthropologie anglo-saxonne (celle de Victor Turner puis de Marshall Sahlins). Il s'agit d'un métier habité par le souci (inculqué et incarné par Arnaldo Momigliano, et aussi par Jonathan Smith) de savoir comment se construisent et se déconstruisent, dans le devenir collectif et la longue durée, nos évidences scientifiques. Les grands écoliers que j’ai évoqués ici ont été mes maîtres occasionnels. J'aimerais y ajouter Angelo Brelich, que je n'ai jamais rencontré, comme je l'ai dit, mais dont la figure dominait l'école italienne

7 "Mythe et histoire chez Mircea Eliade. Réflexions d'un écolier en histoire des religions", Institut national genevois, Annales 1993 (Actes de l'ING 37), 1994, p. 33-49, repris dans Exercices de mythologie, Genève, éditions Labor et Fides, 2004. 
d'histoire des religions quand j'ai eu la chance de passer cette année, bienheureuse, à l'Institut suisse de Rome.

En conclusion, peut-être, faudrait-il dire un mot non plus des personnes, mais des lieux. Les villes: Rome, bien sûr, en premier lieu. Mais aussi Paris, Chicago et Mexico. Avec leurs bibliothèques et leurs Musées, leurs alcools et leurs langues. Athènes (la terrasse de l'institut allemand, rue Phidias, et les concerts de Rebétiko dans la nuit du quartier d'Exarchia...)

Une ferme de montagne en Haute-Savoie, où je rédigeais ma thèse, avec la forêt autour, dont je connaissais chaque arbre. Une île, Céphalonie, avec cette petite maison de pierre et une citerne, dans un verger virgilien.

Et le plus important, à l'origine de tout: les Saintes-Maries-de-la-Mer en 19491950, une cabane de pêcheur, mes premiers souvenirs clairs, d'avant l'exil: braconne dans les enganes (on tendait des collets) et pêche aux coquillages, entre dunes et marais salants.

Philippe Borgeaud

Professeur ordinaire d'histoire des religions antiques

Faculté des Lettres

Université de Genève,

UniBastions

$\mathrm{CH}-1211$ Genève 4

Philippe.Borgeaud@unige.ch 